\title{
Controllability of Structural, Optical and Electrical Properties of Ga doped ZnO Nanowires Synthesized by Physical Vapor Deposition
}

\author{
Sang Yeol Lee ${ }^{\dagger}$ \\ Department of Semiconductor Engineering, Cheongju University, Cheongju 360-764, Korea
}

Received March 5, 2013; Revised March 24, 2013; Accepted April 11, 2013

\begin{abstract}
The control of Ga doping in ZnO nanowires (NWs) by physical vapor deposition has been implemented and characterized. Various Ga-doped $\mathrm{ZnO}$ NWs were grown using the vapor-liquid-solid (VLS) method, with Au catalyst on c-plane sapphire substrate by hot-walled pulsed laser deposition (HW-PLD), one of the physical vapor deposition methods. The structural, optical and electrical properties of Ga-doped ZnO NWs have been systematically analyzed, by changing Ga concentration in $\mathrm{ZnO}$ NWs. We observed stacking faults and different crystalline directions caused by increasing Ga concentration in ZnO NWs, using SEM and HR-TEM. A $D^{0} \mathrm{X}$ peak in the PL spectra of Ga doped ZnO NWs that is sharper than that of pure ZnO NWs has been clearly observed, which indicated the substitution of Ga for $\mathrm{Zn}$. The electrical properties of controlled Ga-doped ZnO NWs have been measured, and show that the conductance of ZnO NWs increased up to $3 \mathrm{wt} \%$ Ga doping. However, the conductance of $5 \mathrm{wt} \%$ Ga doped ZnO NWs decreased, because the mean free path was decreased, according to the increase of carrier concentration. This control of the structural, optical and electrical properties of ZnO NWs by doping, could provide the possibility of the fabrication of various nanowire based electronic devices, such as nano-FETs, nano-inverters, nano-logic circuits and customized nano-sensors.
\end{abstract}

Keywords: ZnO, Nanowire, Doped nanowire

\section{INTRODUCTION}

$\mathrm{ZnO}$ is a promising material, which has transparent, piezoelectric, wide-band gap (3.4 eV) and high exciton binding energy $(60 \mathrm{meV})$, for application in electronics and optoelectronics devices. It has been extensively used for light emitting devices (LEDs), nano generator and laser diodes (LDs) using optical characteristics, such as short wavelength and wide band gap [15]. ZnO has n-type property due to native defects, such as oxygen vacancies and $\mathrm{Zn}$ interstitials. But these characteristics can be varied depending on processing conditions, such as pressure

${ }^{\dagger}$ Author to whom all correspondence should be addressed:

E-mail: sylee@cju.ac.kr

Copyright $\odot 2013$ KIEEME. All rights reserved.

This is an open-access article distributed under the terms of the Creative Commons Attribution Non-Commercial
License (http://creativecommons.org/licenses/by-nc/3.0) which permits unrestricted noncommercial use,

distribution, and reproduction in any medium, provided the original work is properly cited
ditus and temperature, between insulating and conducting properties $[6,7]$. Also, doping is one of the powerful methods to control electrical properties. In particular, doping controls have already been reported to change the behavior from n-type to $\mathrm{p}$-type, by using various doping materials in thin film [8-11]. However, the doping has been limited, due to restrictive volume and native one-dimensional structure in nanostructures [12]. So, many researchers have studied doping problems of nanowires (NWs). Yuan et al. reported a controlled growth and doping process of well-aligned Ga doped ZnO NWs [13]. Also, M. Sakurai et al. reported electrical transfer properties of Ga doped ZnO NWs with NW FET fabrication [14]. Above these works, Ga doped ZnO NWs were fabricated by chemical vapor deposition (CVD) method, which is not so easy to control, and complicated to fabricate NWs [15]. Therefore, we have used the physical vapor deposition (PVD) method, like hot-walled pulsed laser deposition (HWPLD), which is a relatively simple process for the application of electronics and optoelectronics devices, compared with CVD 
[16]. Also, this process can easily be used to adjust the composition of NWs, by controlling the element component of targets. However, there could be a lot of defects and difficulties of uniform doping in fabricated doped NWs by PVD.

In this paper, we have fabricated Ga doped $\mathrm{ZnO}$ NWs with various concentrations (pure, $1 \mathrm{wt} \%, 3 \mathrm{wt} \%$, and $5 \mathrm{wt} \%$ ), by using the HW-PLD method. We observed that the Ga dopants had been successfully controlled in $\mathrm{ZnO}$ crystal lattice, by using optical and electrical measurements. Based on these results, we demonstrate that physical vapor deposition is simple and efficient to fabricate tunable Ga doped $\mathrm{ZnO}$ NWs, and presents systematic control of Ga doping into ZnO NWs.

\section{EXPERIMENTS}

ZnO NWs were fabricated using the vapor-liquid-solid (VLS) mechanism, by a self-designed HW-PLD. Figure 1 shows a schematic diagram of the HW-PLD system. It is very simple and powerful to fabricate the NWs, which have the same material composition as the target. Moreover, this fabrication system does not require any chemical reactions in the chamber. Therefore, it is very easy to fabricate NWs, without any complex source materials and source tool. In order to control the Ga concentration in $\mathrm{ZnO}$ NWs, we used $0 \mathrm{wt} \%, 1 \mathrm{wt} \%, 3 \mathrm{wt} \%$ and $5 \mathrm{wt} \%$ Ga doped $\mathrm{ZnO}$ targets with ball-milling the ceramic powders ( $\mathrm{ZnO}$ and $\mathrm{Ga}_{2} \mathrm{O}_{3}$ ), followed by the isostatic press and sintering process [17]. These targets were irradiated by excimer laser, with the operating condition of $248 \mathrm{~nm}, 1.5 \mathrm{~J} / \mathrm{cm}^{2}$ and $10 \mathrm{~Hz}$. A target holder was rotated at $4 \mathrm{rpm}$ during the deposition, to prevent cone formation, and to ensure uniform ablation of the target. The Au catalyst thin film was deposited $2 \mathrm{~nm}$ on c-plane sapphire substrate by thermal evaporation. Then, $\mathrm{ZnO} \mathrm{NWs}$ were fabricated at $800^{\circ} \mathrm{C}$ for 30 minutes. The NW fabrication pressure was maintained at 1.2 torr. Constant streams of Ar (90 sccm) gases flowed in the furnace during ZnO NWs fabrication. Substrates were located at 2 to $3 \mathrm{~cm}$ from the target. The crystalline direction and morphology of as-grown Ga-doped ZnO NWs were analyzed, by using X-ray diffraction (XRD), field emission scanning electron microscope (FE-SEM) and high resolution transmission electron microscopy (HR-TEM), respectively. The optical properties of Ga-doped ZnO NWs were measured by using photoluminescence (PL), depending on temperature. Also, in order to measure electrical properties, as-grown $\mathrm{ZnO}$ NWs were suspended in isopropyl alcohol, and dispersed on oxidized Si substrates. Finally, electrodes were fabricated, by using conventional photo-lithography process and e-beam lithography. Ti (10 nm) and Au (180 nm) were deposited by using electron beam deposition and thermal evaporation, respectively. Fabricated Ga-doped ZnO NW devices were analyzed by using a semiconductor parameter analyzer.

\section{RESULTS AND DISCUSSION}

Figure 2 shows FE-SEM images of as-grown (a) undoped, (b) 1 $w t \%$, (c) $3 \mathrm{wt} \%$, and (d) $5 \mathrm{wt} \%$ Ga doped ZnO NWs. The diameter and the length of these ZnO NWs were 80 to $100 \mathrm{~nm}$, and approximately $10 \mu \mathrm{m}$, respectively. As shown in Fig. 2, we observed that all of $\mathrm{ZnO}$ NWs had Au particle at the top of NWs. This result clearly indicates VLS growth of ZnO NWs. It is interesting to note that stacking faults have been observed in $1 \mathrm{wt} \%, 3 \mathrm{wt} \%$, and 5 wt\% Ga doped ZnO NWs, different from undoped ZnO NWs. As Ga concentration was increased, the stacking faults also increased, and were much denser, mainly due to the induced stress caused by the different lattice parameters between $\mathrm{Zn}$ and Ga. More detailed analysis will be added later, by using HR-TEM.

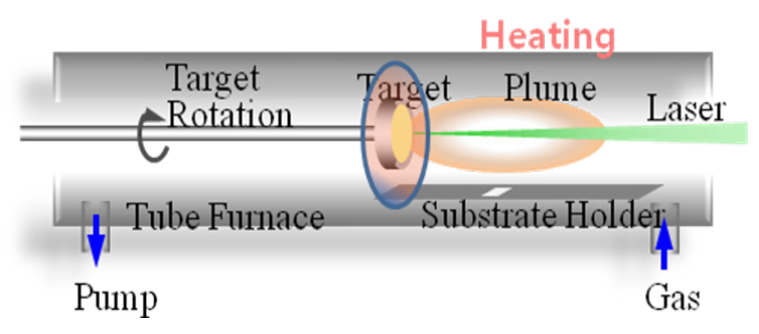

Fig. 1. Schematic diagram of the hot-walled PLD system used in the ZnO NW growths.
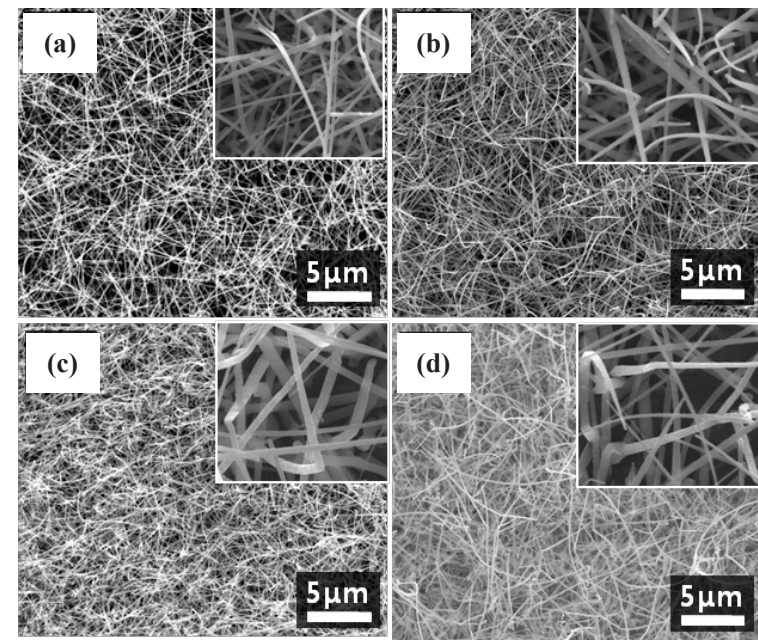

Fig. 2. FE-SEM images of various ZnO-based NWs with (a) undoped, (b) $1 \mathrm{wt} \%$, (c) $3 \mathrm{wt} \%$, and (d) $5 \mathrm{wt} \%$ Ga-doped ZnO NWs. Arrow indicates the Au nano-particle at the end of nanowire. Arrow in the inset indicates Au catalyst droplet at the end of NWs.

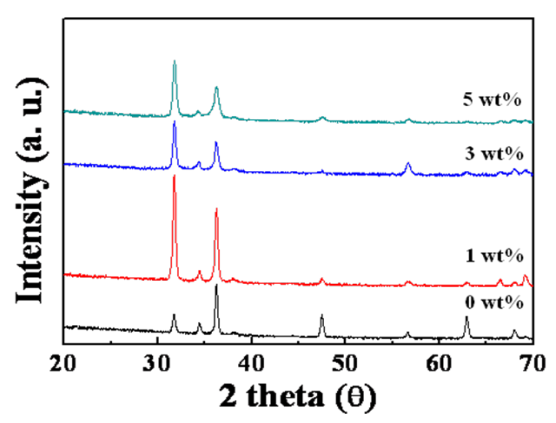

Fig. 3. X-ray diffraction of undoped, $1 \mathrm{wt} \%, 3 \mathrm{wt} \%$ and $5 \mathrm{wt} \% \mathrm{Ga}$ doped ZnO NWs.

We have examined the structural effect of various Ga concentration in $\mathrm{ZnO}$ NWs on the crystallization of $\mathrm{ZnO}$ NWs, using XRD. Figure 3 shows the crystalline direction of (a) undoped (b) $1 \mathrm{wt} \%$, (c) $3 \mathrm{wt} \%$, and (d) $5 \mathrm{wt} \%$ Ga doped ZnO NWs in the XRD pattern. Pure ZnO NWs mainly grew (100), (002) and (101) planes, of which (100) and (101) planes showed the dominant intensity. The absence of the (002) peak in the XRD pattern was explained by the texture of the film, where most of the wires lay on the plane of the substrate [18]. It is interesting to note that the peak of the (002) plane has disappeared gradually, as Ga concentration increased, and the peak intensity of the (100) plane that was weak in pure ZnO NWs has been gradually stronger. Furthermore, as Ga concentration increased, the (002) plane showed 

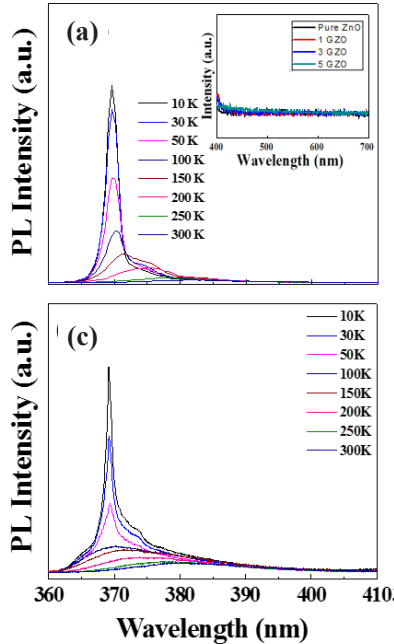

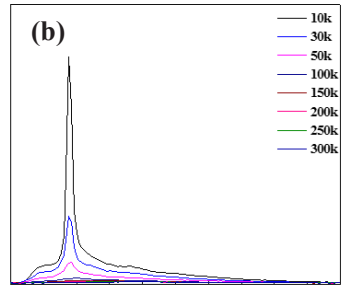

(d)

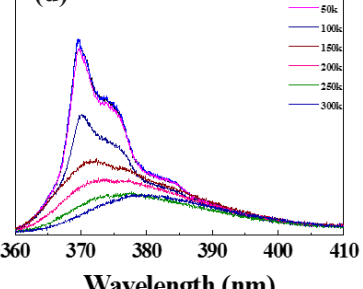

Wavelength (nm)
Fig. 4. Photoluminescence spectra of (a) undoped, (b) 1 wt\%, (c) 3 wt\%, and (d) $5 \mathrm{wt} \%$ Ga-doped $\mathrm{ZnO}$ NWs. The inset shows photoluminescence spectra of the defect range.

a broad peak. The full width at half maximum (FWHM) of the (002) plane of undoped, $1 \mathrm{wt} \%, 3 \mathrm{wt} \%$ and $5 \mathrm{wt} \%$ Ga doped $\mathrm{ZnO}$ NWs has been calculated to be about $0.4^{\circ}, 0.42^{\circ}, 0.66^{\circ}$ and $0.71^{\circ}$, respectively. As shown in SEM images, XRD results also indicated a lattice distortion, due to the introduction of other elements, which had different lattice parameters.

We also investigated the optical properties of $\mathrm{ZnO}$ NWs. Figure 4 shows the PL spectra of (a) undoped, (b) $1 \mathrm{wt} \%$, (c) $3 \mathrm{wt} \%$, and (d) $5 \mathrm{wt} \% \mathrm{Ga}$ doped ZnO NWs, depending on the measurement temperature. The band edge emission of Ga doped $\mathrm{ZnO}$ NWs was shifted in the blue direction, compared with undoped ZnO NWs. These results are ascribed to the Burstein-Moss effect, which occurs at a wider optical band gap, because of the difference of effective mass on increasing carrier concentration $[19,20]$. As shown in the inset of Fig. 4(a), all of the ZnO NWs had dominant band edge emission, without any defects emission. These results indicated that the Ga dopants occupied defect sites in the $\mathrm{ZnO} \mathrm{NW}$ [14]. So, the band edge emission of these $\mathrm{ZnO}$ NWs were sharp, and of high intensity. For $5 \mathrm{wt} \%$ Ga doped ZnO NWs, the band edge emission was showed broad and low intensity. At low temperature of 10K, PL spectra of various Ga doped ZnO NWs have been separated into individual emission peaks. Donor-bound exciton emission was clearly observed at all of the Ga doped ZnO NWs. These results represented successful n-type Ga-doping status in ZnO NWs.

Figure 5 shows the HR-TEM images of (a) undoped, (b) 1 wt\%, (c) $3 \mathrm{wt} \%$, and (d) $5 \mathrm{wt} \%$ Ga doped ZnO NWs. All of the ZnO NWs have been observed to be single crystal-like, by using selectedarea electron diffraction (SAED) patterns. Undoped $\mathrm{ZnO}$ NW has a wurtzite structure grown along the [001] direction, and smooth surface. The growth direction of Ga doped $\mathrm{ZnO}$ NWs has been observed to be changed, by using XRD results. The Ga concentration in $\mathrm{ZnO} \mathrm{NW}$ induced stress between the Ga dopant and the original lattice element. We observed clearly the stacking faults of Ga doped ZnO NWs in Fig. 5. The covalent bond lengths of Ga-O and Zn-O were $1.92 \AA$ and $1.97 \AA$, respectively. So, accumulated stress induced by the difference of the covalent bond lengths between Ga-O and Zn-O causes the stacking faults, in order to relax the induced stress. The density of stacking faults in undoped, 1 $\mathrm{wt} \%, 3 \mathrm{wt} \%$ and $5 \mathrm{wt} \% \mathrm{Ga}$ doped $\mathrm{ZnO}$ NWs were $0 \mathrm{~cm}^{-1}, 3.03 \times 10^{3}$ $\mathrm{cm}^{-1}, 6.67 \times 10^{3} \mathrm{~cm}^{-1}$ and $1.25 \times 10^{4} \mathrm{~cm}^{-1}$, respectively. When the Ga concentration increased in $\mathrm{ZnO}$ NWs, the stacking faults were
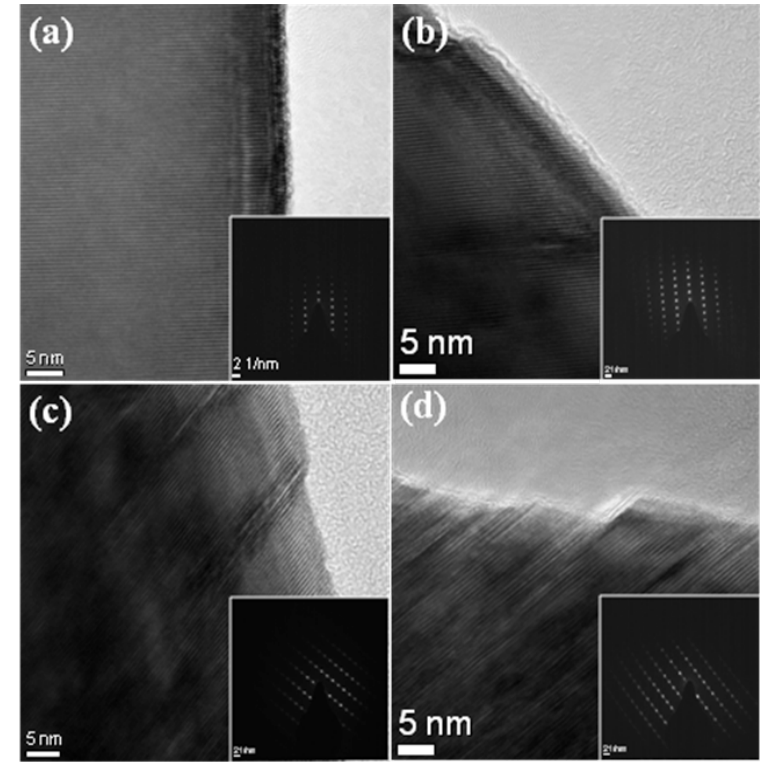

Fig. 5. HR-TEM images of (a) undoped, (b) 1 wt\%, (c) 3 wt\%, and (d) 5 wt\% Ga doped ZnO NWs.

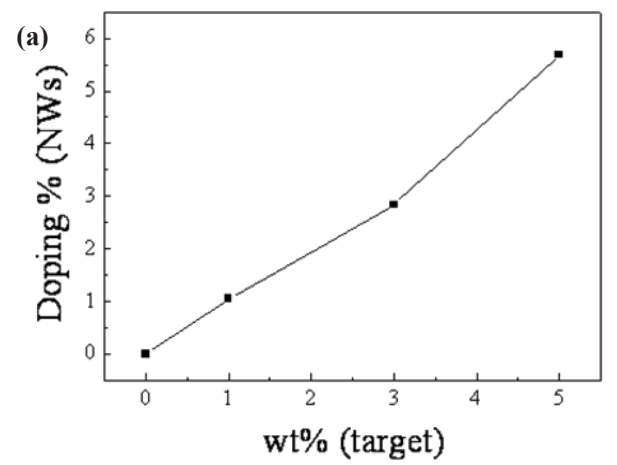

(b)
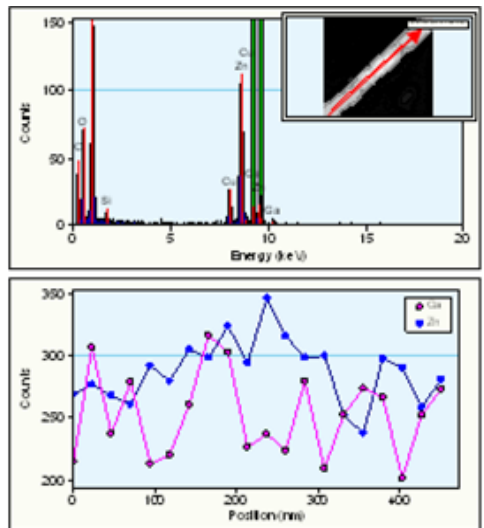

Fig. 6. EDX spectra of (a) undoped, (b) $1 \mathrm{wt} \%$, (c) $3 \mathrm{wt} \%$, and (d) 5 wt\% Ga-doped ZnO NWs.

shorter and denser. In particular, $5 \mathrm{wt} \%$ doped ZnO NWs had a lot of stacking faults, due to strong stress by different covalent bond length.

Also, we measured the EDS spectrum, in order to confirm the Ga ratio in ZnO NWs. As shown in Fig. 6(a), the Ga ratio at undoped, $1 \%, 3 \%$ and $5 \mathrm{wt} \%$ Ga doped ZnO NWs was $0 \mathrm{wt} \%, 1.05$ $w t \%, 2.84 \mathrm{wt} \%$ and $5.69 \mathrm{wt} \%$, respectively. We confirmed that the Ga concentrations in ZnO NWs were successfully controlled by 


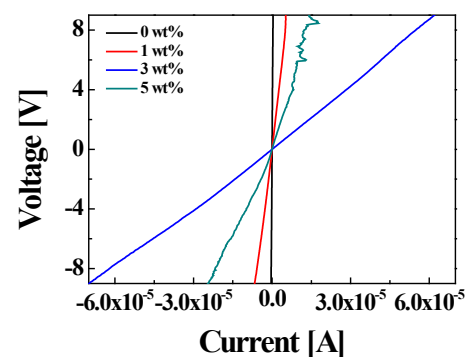

Fig. 7. Typical I-V curve of undoped, 1 wt\%, 3 wt\% and $5 \mathrm{wt} \% \mathrm{Ga}-$ doped ZnO NWs.

the physical vapor deposition method, using different $\mathrm{ZnO}$ targets, with Ga dopants from 0 wt\% to $5 \mathrm{wt} \%$. Figure $6(\mathrm{~b})$ shows the result of the line profile of EDX to confirm uniform distribution of the Ga dopant in $5 \mathrm{wt} \%$ Ga doped $\mathrm{ZnO} \mathrm{NW}$ along the growth direction. It has been confirmed that $\mathrm{Ga}$ dopants into in $\mathrm{ZnO}$ NWs were observed uniformly in all of the positions, and the average mean counts of Ga dopants per position (nm) were about 255.

Electrical properties of undoped $1 \mathrm{wt} \%, 3 \mathrm{wt} \%$ and $5 \mathrm{wt} \% \mathrm{Ga}$ doped ZnO NWs were examined, by using semiconductor parameter analyzer. I-V curves of ZnO NWs, as depicted in Fig. 7, confirmed the resistance variation as increasing Ga concentration in the $\mathrm{ZnO}$ NWs. The resistance of undoped, $1 \mathrm{wt} \%, 3 \mathrm{wt} \%$ and $5 \mathrm{wt} \% \mathrm{Ga}$ doped $\mathrm{ZnO}$ NWs was 22.13 $\mathrm{M} \Omega, 1.30 \mathrm{M} \Omega, 0.13 \mathrm{M} \Omega$ and $0.39 \mathrm{M} \Omega$, respectively. The resistance of Ga doped ZnO NWs from undoped to $3 \mathrm{wt} \%$ clearly decreased by one order. From these results, it could be understood that Ga dopants were successfully doped into $\mathrm{ZnO}$ NWs, resulting in the generation of donor carriers, which reduced the resistance, when the Ga concentration increased up to $3 \mathrm{wt} \%$ in $\mathrm{ZnO}$ NWs. However, $5 \mathrm{wt} \%$ Ga doped $\mathrm{ZnO}$ NW had a lot of Ga concentration, which induced the decrease of mean free path of the carrier [21]. Consequently, it could be suggested that the resistance of heavily doped $\mathrm{ZnO}$ NW increased slightly, due to the increase of scattering. For more detail of the electrical properties, we have attempted to fabricate various Ga doped $\mathrm{ZnO}$ NW FETs. However, it was very hard to observe the off-state in Ga-doped ZnO NW FETs, due to the high doping concentration, resulting in conducting NWs. As a result, we clearly confirmed that the electrical properties of ZnO NWs were systematically controlled by varying the Ga concentration.

\section{CONCLUSIONS}

In summary, we have fabricated controlled Ga doped $\mathrm{ZnO}$ NWs, by using the PVD method. We observed a good single crystalline of ZnO NWs by HR-TEM measurement. The growth direction was changed, according to the increase of Ga concentration. These results agreed well with those of XRD and PL. Ga doped $\mathrm{ZnO}$ NWs clearly showed a $\mathrm{D}^{0} \mathrm{X}$ peak without any defect emission. These results exhibited that Ga dopants in $\mathrm{ZnO}$ NWs were successfully doped. Also, we confirmed that the electrical properties of ZnO NWs were systematically controlled by changing Ga concentration in the $\mathrm{ZnO}$ NWs. Controlled Ga-doping into $\mathrm{ZnO}$ NWs by the physical vapor deposition has been easily achieved, and these various Ga-doped $\mathrm{ZnO}$ NWs could open the possibility of new applications in nanoelectronic and nanosensing devices.

\section{REFERENCES}

[1] Huang, M. H.; Mao, S.; Feick, H.; Yan, H.; Wu, Y.; Kind, H.; Weber, E.; Russo, R.; Yang, P. Science. 2001, 292, 1897 [DOI: http:// dx.doi.org/10.1126/science.1060367].

[2] Yan, H.; Johnson, J.; Law, M.; He, R.; Knutsen, K.; McKinney, J. R.; Pham, J.; Saykally, R.; Yang, P. Adv. Mater. 2003, 15, 1907 [DOI: http://dx.doi.org/10.1002/adma.200305490].

[3] Könenkamp, R.; Word, R.C.; Schlegel, C. Appl. Phys. Lett. 2004, 85, 6004 [DOI: http://dx.doi.org/10.1063/1.1836873].

[4] Wang, H. T.; Kang, B. S.; Ren, F.; Tien, L. C.; Sadik, P. W.; Norton, D. P.; Pearton, S. J.; Lin, J. Appl. Phys. Lett. 2005, 86, 243503 [DOI: http://dx.doi.org/10.1063/1.1949707].

[5] Law, M.; Greene, L. E.; Johnson, J. C.; Saykally, R.; Yang, P. Nature Mater. 2005, 4, 455 [DOI: http://dx.doi.org/10.1038/ nmat1387].

[6] Xiong, G.; Wilkinson, J.; Mischuck, B.; Tuzemen, S.; Ucer, K. B.; Williams, R. T. Appl. Phys. Lett. 2002, 70.1195.

[7] Ma, Y.; Du, G. T.; Yang, S. R.; Li, Z. T.; Zhao, B. J.; Yang, X. T.; Yang, T. P.; Zhang, Y. T.; Liu, D. L. J. Appl. Phys. 2004, 95, 6268.

[8] Jiang, X.; Wong, F. L.; Fung, M. K.; Lee, S. T. Appl. Phys. Lett. 2003, 83, 1875 [DOI: http://dx.doi.org/10.1063/1.1605805].

[9] Yu, Z. G.; Wu, P.; Gong, H. Appl. Phys. Lett. 2006, 88, 132114 [DOI: http://dx.doi.org/10.1063/1.2174089].

[10] Kang, H. S.; Ahn, B. D.; Kim, J. H.; Kim, G. H.; Lim, S. H.; Chang, H. W.; Lee, S. Y. Appl. Phys. Lett. 2006, 88, 202108 [DOI: http:// dx.doi.org/10.1063/1.2197317].

[11] Kim, K.; Debnath, P. C.; Park, D.-H.; Kim, S. S.; Lee, S. Y. Appl. Phys. Lett. 2010, 96, 083103 [DOI: http://dx.doi. org/10.1063/1.3290247].

[12] Zou, C. W.; Gao, W. Trans. Electr. Electron. Mater. 2010, 11, 1 [DOI: http://dx.doi.org/10.4313/TEEM.2010.11.1.001].

[13] Yuan, G. D.; Zhang, W. J.; Jie, J. S.; Fan, X.; Tang, J. X.; Shafiq, I.; Ye, Z. Z.; Lee, C. S.; Lee, S. T. Adv. Mater. 2008, 20, 168 [DOI: http://dx.doi.org/10.1002/adma.200701377].

[14] Sakurai, M.; Wang, Y. G.; Uemura, T.; Aono, M. Nanotechnology. 2009, 20, 155203 [DOI: http://dx.doi.org/10.1088/09574484/20/15/155203].

[15] Terasako, T.; Shirakata, S. Jpn. J. Appl. Phys. 2005, 44, L1410L1413 [DOI: http://dx.doi.org/10.1143/JJAP.44.L1410].

[16] Lee, S. Y.; Song, Y. W.; Jeon, K. A. J. Cryst. Growth. 2008, 310, 4477 [DOI: http://dx.doi.org/10.1016/j.jcrysgro.2008.07.041].

[17] Song, Y. W.; Lee, S. Y. Thin Solid Films. 2008, 518, 1323.

[18] Goris, L.; Noriega, R.; Donovan, M.; Jokisaari, J.; Kusinski, G.; Salleo, A. J. Electron. Mater. 2009, 38, 586 [DOI: http://dx.doi. org/10.1007/s11664-008-0618-x].

[19] Burstein, E. Phys. Rev. 1954, 93, 632 [DOI: http://dx.doi. org/10.1103/PhysRev.93.632].

[20] Moss, T. S. Proc. Phys. Soc. Lond. 1954, B 67, 775.

[21] Zhong, J.; Malcolm, S. G. Nano. Lett. 2006, 6, 128-132 [DOI: http://dx.doi.org/10.1021/nl062183e]. 